\title{
The effect of aerobic exercise training on leptin and pulmonary function tests during weight loss in men with visceral obesity
}

\author{
Mohammad Parastesh $^{1 * \mathbb{D}}$, Esmaeil Alibakhshi $^{2,3}{ }^{\circledR}$, Abbas Saremi $^{\circledR}$, Negar Shavandi $^{(\mathbb{D}}$ \\ ${ }^{1}$ Department of Sports physiology, Faculty of Sports Sciences, Arak University, Arak, Iran. \\ ${ }^{2}$ Pulmonology Department, Baqyiatallah Hospital, Baqyiatallah University of Medical Science, Tehran, Iran. \\ ${ }^{3}$ Parc Sanitari Sant Joan de Deu, Faculty of Medicine, Barcelona University, Barcelona, Spain. \\ ${ }^{4}$ Department of Biology, Faculty of Sciences, York University, Toronto, Canada.
}

*Corresponding Author: Mohammad Parastesh, Assistant Professor, Department of Sports Physiology, Faculty of Sports Sciences, Arak University, Arak, Iran , Tel: 09331528384, Email: M-parastesh@Araku.ac.ir

\begin{abstract}
Background and aims: It has recently been documented that obesity is a global problem in all age groups, so that it affects about 250 million people in the world, accounting for $7 \%$ of the world's population. Adipose tissue has an important role in inducing lung dysfunction through production and secretion of adipokines such as leptin. The aim of this study was to investigate the effect of a period of aerobic training on lung function indices and serum leptin levels in obese men.

Methods: A total of 23 untrained obese men in Arak were recruited voluntarily for the study and randomly assigned to obese aerobic training group (Ob-AT, $\mathrm{n}=12)\left(45.26 \pm 2.45\right.$ years, body mass index $(\mathrm{BMI})$ of $\left.31.81 \pm 0.77 \mathrm{~kg} / \mathrm{m}^{2}\right)$ and obese control group $(\mathrm{Ob}-\mathrm{Cnt}, \mathrm{n}=11)$ $(47.03 \pm 3.36$ years, BMI of $31.45 \pm 0.96 \mathrm{~kg} / \mathrm{m} 2)$. Another normal control group (No-Cnt, $n=9)$ was sampled from individuals with normal weight to compare at the base level $\left(45.1 \pm 4.1\right.$ years, BMI of $\left.24.63 \pm 0.45 \mathrm{~kg} / \mathrm{m}^{2}\right)$. The obese exercise group participated in 12 weeks of progressive aerobic training, 3 sessions a week, 1.5 hours per session. Body composition (CT scan), leptin levels and lung function were measured before and after exercise protocol.

Results: The participants with normal weight had significantly lower leptin concentration $(P=0.01)$ and a higher lung function $(P=0.016)$ compared to the obese participants. Moreover, the results indicated that leptin and obesity had an inverse correlation with lung function indices (forced vital capacity [FVC] and forced expiratory volume in one second $\left.\left[\mathrm{FEV}_{1}\right]\right)(P \leq 0.05)$. After the aerobic activity, obesity (BMI, WHR, body fat) and lung function indices ( $F V C$ and $\mathrm{FEV}_{1}$ ) significantly decreased in the obese aerobic training group compared to the obese control group $(P \leq 0.05)$. In contrast, leptin concentration significantly decreased after the aerobic exercise protocol $(P=0.018)$.

Conclusion: We found that leptin level decreased after 12 weeks of aerobic exercise in obese men and this decrease was associated with the increased lung function and profound changes in body fat. The obtained data suggest that 12 -week aerobic exercise causes alterations in leptin level that plays a key role in improving lung function.

Keywords: Aerobic training, Serum leptin, Lung function, Obese men
\end{abstract}

Received: 14 November 2019, Accepted: 25 April 2020, ePublished: 29 April 2020

\section{Introduction}

Recently, it has been documented that obesity is a worldwide problem in all age groups that affects about 250 million people in the world, accounting for $7 \%$ of total world population. The prevalence of overweight is about twice or three times as much as stated quantity (1). According to the reports of the World Health Organization (WHO), the number of obese and overweight people was around 1.5 billion in 2015 worldwide (2). Obviously, obesity is increasing in various countries. According to the Organization for Economic Cooperation and Development (OECD) projections, the rate of obesity in the United States, Mexico, and the United Kingdom would increase to $47 \%, 39 \%$, and $35 \%$ in 2030 , respectively (3). Abdominal obesity is widespread in several cities of Iran which is estimated that this prevalence is higher compared to Tehran province (76\%), Rafsanjan (54.7\%), Esfahan (84.6\%), Arak (66.8\%), Najafabad (82.2\%) and Mazandaran province (82.2\%) (4). One of the most important reports on the status of obesity in Iran shows that during the years 1999-2007, the prevalence of obesity in 25-64-year-old people increased from $13.6 \%$ to $22.3 \%$ (5).

Interestingly, it was reported that one of the negative impacts of obesity is lung function disorders (6). Additionally, an association was observed between

(C) 2020 The Author(s); Published by Shahrekord University of Medical Sciences. This is an open-access article distributed under the terms of the Creative Commons Attribution License (http://creativecommons.org/licenses/by/4.0), which permits unrestricted use, distribution, and reproduction in any medium, provided the original work is properly cited. 
inflammation and reduced function of lung in obese people. Therefore, it seems that inflammation causes a reduction in lung function (7). Leptin is a $16 \mathrm{U}$ nonglycosylated polypeptide, which is produced by deposits and exists in the blood circulation system. Leptin seems to be in the regulation of energy balance, modulation of inflammation response, and mediation of immune function (8). Leptin has a pro-inflammatory effect and enhances the production of pro-inflammatory factors such as tumor necrosis factor-alpha (TNF $\alpha$ ) and interleukin-1 (IL-1) (9). It was reported that leptin probably causes reduced lung function in obese people through inflammation. Briefly, reduced lung function results from a high level of leptin and inflammation (10). In the study by Shareef et al, there was a high correlation between pulmonary function indexes (including forced vital capacity [FVC] and forced expiratory volume in one second [FEV1]) and serum leptin levels. This study suggested that the increase in serum leptin was associated with a decrease in pulmonary function indexes (11). There was also a significant correlation between the increase in serum leptin levels and chronic obstructive pulmonary disease (COPD) in the study conducted by Sood (12). However, in various studies, the mechanism for that is not clear and different studies reported inconsistent results. In the study conducted by Kim et al, serum leptin levels had a significant relationship with FEV1, but not with FVC and FEF $75 \%$ and PC20 in all subjects (13). Lack of physical exercise is one of risk factors which resulted in obesity and inflammation status (14). Heiniger et al demonstrated that body mass index (BMI) was used to classify patients in earlier studies. This is not consistently appropriate because patients can have significant muscle wasting without a significant decrease in total body weight if there is a corresponding increase in fat mass. BMI, although known to be associated with mortality and body composition parameters, is not the predictor of the outcome. BMI and body composition are severely affected, with a decline of fat mass (FM) and especially fat-free mass (FFM), and inversely correlated with the severity of emphysema and clinical outcomes, thus increasing diseaserelated morbidity and mortality (15). The main purpose of this study was to investigate whether physical exercise could reduce adipose tissue mass and reduced leptin level could improve lung function in obese people.

\section{Materials and Methods \\ Study design}

The study has been approved by the Ethics Committee and Research Committee at the Faculty of Sports Science, Arak University. A total of 23 middle-aged and elderly obese men with MRC dyspnea score $\geq 3$ were included in the study. All subjects participated in this study were assessed in terms of general characteristics (sex, age, weight, height, and BMI) and informed written consent was signed by the candidates for the study after receiving full information on the objectives, techniques, and possible consequences prior to the study. The subjects were randomly assigned to two groups as follows: obese control group of $11 \mathrm{men}$ $\left(47.03 \pm 3.36\right.$ years old, BMI: $\left.31.45 \pm 0.96 \mathrm{~kg} / \mathrm{m}^{2}\right)$ and physical exercise group of 12 men $(45.26 \pm 2.45$ years, BMI: $31.81 \pm 0.77 \mathrm{~kg} / \mathrm{m}^{2}$ ). The sample size was calculated based on previous studies using $\mathrm{G}^{*}$ Power software, with an effect size of 1.2 , error type $1(\alpha=0.5)$, and error type 2 (Power=0.85). Physical exercise group participated in an aerobic exercise protocol for 12 weeks whereas obese control group had their normal lifestyle. Additionally, a group of normal men $(45.1 \pm 4.1$, BMI: $24.63 \pm 0.45 \mathrm{~kg} /$ $\mathrm{m}^{2}$ ) were included in the study to compare their results with those of the obese group. Moreover, the participants were ensured that all their data and information would be kept confidential and that they would be used for research purposes.

\section{Inclusion criteria}

Inclusion criteria for the study were as follows: BMI $\geq 30$ $\mathrm{kg} / \mathrm{m}^{2}$ and little daily exercise (less than 30 minutes per day according to physical exercise questionnaire) (16), the stability of cardiorespiratory function (at least two months without changes in semiotics) demonstrative of moderate (predicted $50 \% \leq \mathrm{FEV} 1<80 \%$ ), severe (predicted $30 \% \leq \mathrm{FEV} 1<50 \%)$ obstruction, continual symptoms, limited physical activity, frequent exacerbations, frequent night-time symptoms, FEV $1 \leq 60 \%$ of predicted, PEFR variability of $>60 \%$, and written informed consent signed by candidates for the study after receiving full information on the objectives, techniques and possible consequences of the consent.

\section{Exclusion criteria}

Exclusion criteria were any treatment interventions that affected laboratory results including smoking, cardiovascular arrhythmia, cardiac ischemia during the exercise test, cardiac surgery during the last 3 months, neuromuscular and orthopedic disorders, rheumatoid arthritis, metabolic syndrome that could interfere with the results or make it difficult to obtain super performance, severe malnutrition, treatment with drugs having a potential effect on the muscle structure and function (e.g. corticosteroids), ethical reasons and intensive physical activities that caused disorder in blood pressure and heart rate.

\section{Aerobic exercise training protocol}

An introductory session was held to inform the participants of safety tips and instruction about treadmill use. An exercise physiology expert was employed to supervise all exercise sessions. Exercise sessions were held in Sports Science Laboratory, Arak University. The aerobic exercise protocol was based on the American College of Sports Medicine (ACSM) recommendations for middle-aged obese men (17). The exercise was performed according to 60\%-65\% 
maximum heart rate during first week that was continued for 25-30 minutes. At the end of the sixth week, it was done at $65 \%-70 \%$ maximum heart rate which continued for 35-40 minutes. At the end of the seventh week, it was done at $70-75 \%$ maximum heart rate which continued for 50-55 minutes. At the end of the 12 th week, it was done at 75-80\% maximum heart rate for 50-55 minutes. Each exercise session consisted of 10 minutes for warming, aerobic exercise, and 15 minutes for tension exercise, situps, push-ups, pull-ups, and finally cooling. The physical exercise protocol was performed 3 days a week (Saturday, Monday, and Wednesday) for 12 weeks.

\section{Body composition}

Height $(\mathrm{cm})$ and weight $(\mathrm{kg})$ were measured to calculate BMI using the following formula: weight $(\mathrm{kg}) /$ height $^{2}$ (m). Loin periphery was measured, which is the distance from the narrowest point between pelvis and last costae. Total abdominal adipose tissue, visceral adipose tissue, and hypodermic adipose tissue were measured using CT scan images that were taken at $110 \mathrm{kvp}$. The participants were placed in a sitting position with their hands up and their back in front of the scanner, and L4 and L5 vertebrae were scanned. Then, the levels of total abdominal adipose tissue, visceral adipose tissue, and hypodermic adipose tissue were analyzed using FAT Scan software. Studies showed that CT scan is a precise method to evaluate abdominal obesity (18). Each part of the examination lasted for 12 minutes. This examination was conducted before and after 12 weeks of physical exercise.

\section{Lung function}

According to American Thoracic Society (ATS), European Respiratory Society (ERS), and Global Initiative for Chronic Obstructive Lung Disease (GOLD) criteria, the classification of the subjects was as follows: mild (predicted FEV1<80\%), moderate (predicted 50\% < FEV1 < 80\%), severe (predicted $30 \% \leq \mathrm{FEV} 1<50 \%$ ) and very severe (predicted FEV1 < 30\%) obstruction, and in this study, mild and moderate levels were approved. In addition, insufficient FEV1 / FVC results from multiple coughs, high exhalation volume, and a $200 \mathrm{ml}$ difference between the initial and final FEV1 values. In addition, FEV1 / FVC 70\%; Spirometry tests are acceptable in all research samples. The participants performed this test in sitting position. When a difference of more than $100 \mathrm{~mL}$ was seen, the third test was performed. Tests were performed by an experienced expert and necessary calibration was done (19).

\section{Laboratory measurements}

Blood sampling was conducted twice, the first time before the first exercise session and the second time 48 hours after the last exercise session (after 10-12 hours of fasting at 6-7 am). The samples were centrifuged for 20 minutes at
$3000 \mathrm{rpm}$ and the extracted serum was stored at $-70^{\circ} \mathrm{C}$. The serum concentration of leptin (Bio vendor, Germany) was measured using ELISA method. Within-groups and between-group variance coefficients were less than $7 \%$.

\section{Statistical analysis}

Data were expressed as mean \pm SD. Shapiro-Wilk test was used to confirm the normal distribution of the data. Furthermore, to make a more comprehensive assessment of hypotheses, Pearson correlation was used. The independent $t$ test was used to compare the differences between groups and within groups. All analyses were performed using SPSS version 19.00 and statistical significance was considered to be $P \leq 0.05$.

\section{Results}

The data about body composition and lung function before and after 12 weeks of physical exercise were shown in Table 1 . In the pre-test, no significant difference was observed between obese aerobic training and obese control groups in terms of body weight $(P=0.698)$, BMI $(P=0.589)$, waist size $(P=0.058)$, total abdominal fat $(P=0.083)$, and visceral fat $(P=0.092)$ in the training group in the pre-test. Moreover, no significant differences were observed in the pre-test and post-test in the obese control group in body weight $(P=0.858)$, BMI $(P=0.959)$, waist size $(P=0.768)$, total abdominal fat $(P=0.853)$, and visceral fat $(P=0.992)$. FVC $(P=0.04)$ and $\mathrm{FEV}_{1}(P=0.035)$ indices were higher in normal participants than in obese participants. Serum leptin levels in the pre-test $(P=0.001)$ and post-test $(P=0.001)$ were higher in obese control group than in the normal control group. Additionally, after 12 weeks of aerobic exercise training, serum leptin levels were lower in aerobic training obese group than in the obese control group $(P=0.001)$ (Figure 1$)$. Basically, leptin level had a negative correlation with FEV1 $(r=-0.73, P=0.023)$ and FVC $(\mathrm{r}=-0.60, P=0.018)$ in all subjects (Table 2$)$.

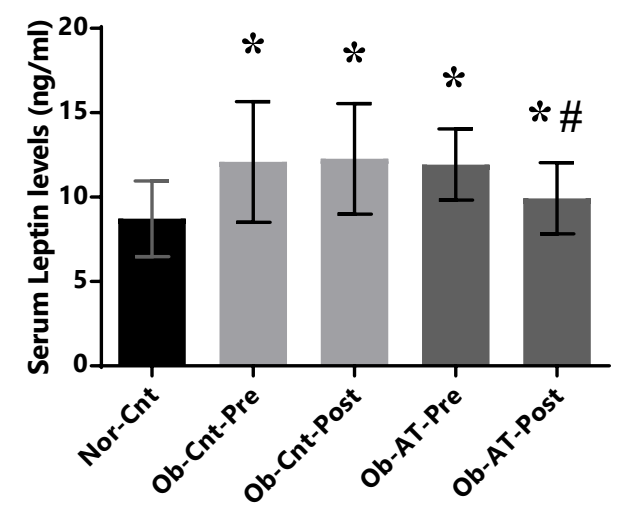

Figure 1. Changes in serum leptin levels in different study groups. Normal control group (Nor-Cnt), Obese control group (Ob-Cnt) and obese aerobic training group (Ob-AT). *: the significant difference with Nor-Cnt $(P<0.05), \#$ : the significant difference between Ob-Cnt and Ob-ET in posttest $(P<0.05)$ 
Table 1. Body composition and pulmonary function of the subjects before and after the exercise protocol

\begin{tabular}{|c|c|c|c|c|c|c|c|}
\hline \multirow{2}{*}{ Groups } & \multirow[t]{2}{*}{ Normal control } & \multicolumn{2}{|c|}{ Obese control } & \multicolumn{2}{|c|}{ Obese aerobic e training } & \multirow{2}{*}{$\begin{array}{c}P \text {-value } \\
\text { (between-group } \\
\text { Comparison in post- } \\
\text { test) }\end{array}$} & \multirow{2}{*}{$\begin{array}{c}P \text {-value } \\
\text { (within-group } \\
\text { comparison in training } \\
\text { group) }\end{array}$} \\
\hline & & Pre-test & Post-test & Pre-test & Post-test & & \\
\hline \multicolumn{8}{|c|}{ Body composition } \\
\hline Body weight (kg) & $78.77 \pm 6.32^{*}$ & $110.27 \pm 6.66$ & $111.64 \pm 6.74$ & $110.25 \pm 7.61$ & $100.79 \pm 7.01 \dagger$ & 0.001 & 0.001 \\
\hline BMI (kg/m²) & $24.63 \pm 0.40^{*}$ & $31.45 \pm 0.96$ & $31.61 \pm 0.99$ & $31.81 \pm 0.77$ & $28.68 \pm 0.75 t$ & 0.001 & 0.001 \\
\hline Waist size $(\mathrm{cm})$ & $88.55 \pm 8.57^{*}$ & $121.09 \pm 10.92$ & $121.45 \pm 11.45$ & $126.66 \pm 11.69$ & $123.00 \pm 11.52$ & 0.02 & 0.2 \\
\hline Visceral fat $\left(\mathrm{cm}^{2}\right)$ & $214.77 \pm 18.14^{*}$ & $250.45 \pm 19.40$ & $250.81 \pm 19.49$ & $256.91 \pm 21.59$ & $233.91 \pm 20.95+$ & 0.005 & 0.045 \\
\hline Skinfold $\left(\mathrm{cm}^{2}\right)$ & $178.78 \pm 12.17^{*}$ & $211.81 \pm 16.17$ & $213.18 \pm 16.90$ & $212.66 \pm 18.46$ & $211.91 \pm 18.04$ & 0.42 & 0.125 \\
\hline Total abdominal fat $\left(\mathrm{cm}^{2}\right)$ & $393.55 \pm 14.67^{*}$ & $462.27 \pm 34.77$ & $464.00 \pm 35.95$ & $459.58 \pm 39.87$ & $455.83 \pm 38.79$ & 0.005 & 0.48 \\
\hline \multicolumn{8}{|c|}{ Pulmonary function } \\
\hline FVC (L) & $4.02 \pm 0.36^{*}$ & $3.83 \pm 0.40$ & $3.85 \pm 0.40$ & $3.89 \pm 0.44$ & $3.99 \pm 0.40 \dagger$ & 0.04 & 0.044 \\
\hline FEV1 (L) & $3.43 \pm 0.28^{*}$ & $3.24 \pm 0.33$ & $3.25 \pm 0.32$ & $3.29 \pm 0.34$ & $3.36 \pm 0.32 \dagger$ & 0.041 & 0.048 \\
\hline FEV1/ FVC(\%) & $85.33 \pm 0.62 *$ & $84.5 \pm 0.22$ & $84.4 \pm 0.11$ & $84.53 \pm 0.34$ & $85.2 \pm 0.21$ & 0.43 & 0.44 \\
\hline
\end{tabular}

Data were expressed as mean \pm standard deviation.

BMI: body mass index. FVC: forced vital capacity, FEV1: the forced expiratory volume in one second.

$* P<0.05$ : significant difference between control normal weight and control obesity.

$+P<0.05$ : significant difference in obese aerobic exercise training group in the pre and post-test.

Table 2. Correlation between lung function indices, leptin, and body composition indices in all subjects $(n=32)$

\begin{tabular}{lcccc}
\hline & \multicolumn{2}{c}{ FEV $_{\mathbf{1}}$} & \multicolumn{2}{c}{ FVC } \\
\cline { 2 - 5 } & $\boldsymbol{r}$ & $\boldsymbol{P}$ value & $\boldsymbol{r}$ & $\boldsymbol{P}$ value \\
\hline Weight & -0.55 & 0.001 & -0.49 & 0.004 \\
BMI & -0.49 & 0.02 & -0.48 & 0.02 \\
Waist size & -0.46 & 0.008 & -0.41 & 0.01 \\
Visceral fat & -0.62 & 0.000 & -0.65 & 0.001 \\
Skinfold & -0.39 & 0.02 & -0.35 & 0.04 \\
Total abdominal fat & -0.55 & 0.001 & -0.49 & 0.004 \\
Leptin & -0.73 & 0.023 & -0.60 & 0.018 \\
\hline
\end{tabular}

FVC: Forced vital capacity, FEV1: The forced expiratory volume in one second

\section{Discussion}

In the present study, we discovered that obesity is associated with lung dysfunction, which consequently leads to an increased serum leptin level. The physical exercise protocol caused an improvement in lung function and a decrease in leptin level. There was a negative correlation between obesity and lung function. Low FVC and FEV 1 in the obese group were seen which are indications of obstructive pulmonary diseases (20). Decreased function of the respiratory system was seen in obese group. Table 2 shows that there is a negative correlation between lung function and obesity indices. These results confirm epidemiologic findings and support hypotheses stating that obesity is one of the risk factors for asthma and respiratory system disorders. Shore and Johnston reported that an increasing body of data including 30 cross-sectional studies and 3 longitudinal studies support the hypothesis that there is a high prevalence of asthma in obese people (21). A recent study showed that there was a strong association between adipose tissue distribution and lung function. Accordingly, abdominal obesity had stronger relationships compared to other risk factors of lung dysfunction (e.g., BMI) (22).
This relationship is due to mechanical effects of obesity or metabolic effects of adipose tissue (23). Abdominal fat decreases total respiratory system compliance, and increases lung bronchial resistance. (24). In obese people, adipokines secreted from adipose tissue (particularly abdominal adipose tissue) may cause lung problems. Adipose is an active endocrine tissue. This tissue has a positive relationship with IL-6, TNF- $\alpha$, and leptin levels (23). It was reported that obesity may result in chronic lung inflammation. In addition, people with symptoms of chronic bronchitis and COPD patients have higher levels of inflammation markers such as IL-1 and TNF- $\alpha$ (25). Other investigation showed that increased inflammation level due to leptin production has a reverse association with $\mathrm{FVC}$ and $\mathrm{FEV}_{1}$. It seems that inflammation factors cause respiratory system diseases (26). Our study showed that leptin levels were higher in obese participants than in normal participants and therefore we can argue that obesity may be related to inflammation and decreased lung function (27). In line with other surveys, we also observed that a higher level of leptin was related to decreased FVC and $\mathrm{FEV}_{1}$, indicating a reverse interrelation (23-25). These findings were confirmed by Sierra-Johnson et al who reported that obesity was related to inflammations and reduced function of respiratory system (26). It was also obvious that after 12-week physical exercise, inflammation status was improved in obese participants, which may be related to better function of lung and respiratory system. It was also reported that there is a reverse relationship between leptin level and impairment of lung function (28). There is a linear relationship between increased leptin levels and increased adiposity (29). Vogiatzis reported that body composition was examined in two trials and an increase in total lean body mass and a reduction in total fat percentage were observed after 12 weeks of progressive resistance exercise. The lack of correlation between peripheral muscle strength and FEV1 is justified because while the muscle 
assessment can be used to measure the systemic limitation, the airway obstruction is used to quantify the impairment of pulmonary function. It thus appears that the degree of impaired lung function does not express a limited peripheral muscle (30). According to these findings, weight loss may cause a reduction in leptin level. In contrast, our investigation showed that an aerobic exercise plan gradually affects inflammation status by decreasing adipose tissue. Consequently, respiratory system diseases improve in obese people. In summary, we can recommend that short-term physical exercise (about 12 weeks) is necessary to decrease adipose tissue mass (particularly abdomen adipose tissue), and the most important achievement of this progress is that leptin levels may decrease. The central role of increased leptin in obese people implies that obese people should have an aerobic exercise protocol to complete weight loss, improve leptin level and increase lung function. These findings suggested that improvement in obesity indices and leptin level due to physical exercise may cause better respiratory system function. Future endeavors seeking to elucidate, validate, and implement improvement in physical exercise advantages through precise mechanisms of physical exercise advantages will likely require extensive investigation to understand inflammation reaction and respiratory system function. The main limitation of the study is the timing of the study protocol (3 times per week, during 12 weeks). More clinical tests are required since participants may not be able to participate in all of the test sessions in the study protocol which results in missing data in the final report. Moreover, we could not control the protocols in their home and workplace.

\section{Conclusion}

In general, the findings of the current study suggest that the twelve-week aerobic exercise program appears to reduce leptin inflammation and increase lung function in people with abdominal obesity while reducing obesity. Although these results suggest that changes in physical activity in obesity and leptin levels may be related to the beneficial effects of physical activity on lung function, further studies are needed to determine the beneficial effects of exercise on inflammatory markers and pulmonary function.

\section{Conflicts of Interest}

None.

\section{Ethical Approval}

The study protocol was approved by the Ethics Committee of Arak University of Medical Sciences (IR.ARAKMU.REC.1398.145). All participants provided informed consent to participate in the study.

Authors Contribution

All authors contributed in preparing this article.

\section{Acknowledgement}

The present research is based on the research project approved by the Applied Research Center of the Islamic Republic of Iran's
PoPolicorce (grant No: 33/04/41/1) at National Elites Foundation, Iran. Additionally, the authors declare their gratitude to all those who assisted us in conducting this study.

\section{References}

1. Rachmi CN, Li M, Alison Baur L. Overweight and obesity in Indonesia: prevalence and risk factors-a literature review. Public Health. 2017;147:20-9. doi: 10.1016/j.puhe.2017.02.002.

2. Mendy VL, Vargas R, Cannon-Smith G, Payton M. Overweight, obesity, and extreme obesity among mississippi adults, 20012010 and 2011-2015. Prev Chronic Dis. 2017;14:E49. doi: 10.5888/pcd14.160554.

3. Mehrabani J, Khazraei Ganjifar Z. Overweight and obesity: a brief challenge on prevalence, complications and physical activity among men and women. MOJ Womens Health. 2018;7(1):19-24. doi: 10.15406/mojwh.2018.07.00161.

4. Jafari-Adli S, Jouyandeh Z, Qorbani M, Soroush A, Larijani B, Hasani-Ranjbar $S$. Prevalence of obesity and overweight in adults and children in Iran; a systematic review. J Diabetes Metab Disord. 2014;13(1):121. doi: 10.1186/s40200-0140121-2.

5. Khabazkhoob $M$, Emamian $M H$, Hashemi $H$, Shariati $M$, Fotouhi A. Prevalence of overweight and obesity in the middleage population: a priority for the health system. Iran J Public Health. 2017;46(6):827-34.

6. Forno E, Han YY, Mullen J, Celedón JC. Overweight, obesity, and lung function in children and adults-a meta-analysis. J Allergy Clin Immunol Pract. 2018;6(2):570-81.e10. doi: 10.1016/j.jaip.2017.07.010.

7. La Cava A. Leptin in inflammation and autoimmunity. Cytokine. 2017;98:51-8. doi: 10.1016/j.cyto.2016.10.011.

8. Procaccini C, La Rocca C, Carbone F, De Rosa V, Galgani M, Matarese G. Leptin as immune mediator: Interaction between neuroendocrine and immune system. Dev Comp Immunol. 2017;66:120-9. doi: 10.1016/j.dci.2016.06.006.

9. Lee SW, Rho JH, Lee SY, Kim JH, Cheong JH, Kim HY, et al. Leptin protects rat articular chondrocytes from cytotoxicity induced by TNF- $\alpha$ in the presence of cyclohexamide. Osteoarthritis Cartilage. 2015;23(12):2269-78. doi: 10.1016/j. joca.2015.06.005.

10. Nasiri Kalmarzi R, Ataee P, Mansori M, Moradi G, Ahmadi $S$, Kaviani Z, et al. Serum levels of adiponectin and leptin in asthmatic patients and its relation with asthma severity, lung function and BMI. Allergol Immunopathol (Madr). 2017;45(3):258-64. doi: 10.1016/j.aller.2016.09.004.

11. Shareef F, Carr TF, Billheimer D, Ledford J, Kraft M. Inverse relationship between leptin and lung function. Am J Respir Crit Care Med. 2017;195:A7453

12. Sood A. Obesity, adipokines, and lung disease. J Appl Physiol (1985). 2010;108(3):744-53. doi: 10.1152/ japplphysiol.00838.2009.

13. Kim KW, Lee KE, Kim ES, Song TW, Park JY, Sohn MH, et al. Association between Leptin and Asthma in Children. Pediatric Allergy Respiratory Disease. 2006;16(4):287-95.

14. Laursen TL, Zak RB, Shute RJ, Heesch MWS, Dinan NE, Bubak MP, etal. Leptin, adiponectin, and ghrelin responses to endurance exercise in different ambient conditions. Temperature (Austin). 2017;4(2):166-75. doi: 10.1080/23328940.2017.1294235.

15. Heiniger S, Viswanathan B, Gedeon J, Paccaud F, Bovet P. Trends in prevalence, awareness, treatment and control of high blood pressure in the Seychelles between 1989 and 2013. J Hypertens. 2017;35(7):1465-73. doi: 10.1097/ hjh.0000000000001358.

16. Wanner M, Probst-Hensch N, Kriemler S, Meier F, Bauman A, Martin BW. What physical activity surveillance needs: 
validity of a single-item questionnaire. $\mathrm{Br} \mathrm{J}$ Sports Med. 2014;48(21):1570-6. doi: 10.1136/bjsports-2012-092122.

17. Riebe D, Franklin BA, Thompson PD, Garber CE, Whitfield GP, Magal $M$, et al. Updating ACSM's recommendations for exercise preparticipation health screening. Med Sci Sports Exerc. 2015;47(11):2473-9. doi: 10.1249/mss.0000000000000664.

18. Leone N, Courbon D, Thomas F, Bean K, Jégo B, Leynaert B, et al. Lung function impairment and metabolic syndrome: the critical role of abdominal obesity. Am J Respir Crit Care Med. 2009;179(6):509-16. doi: 10.1164/rccm.200807-1195OC.

19. Shavandi N, Saremi A, Moeeni L, Parastesh M, Ghorbani A, Heidarpoor RA. The comparison of the responses of lung function indices to aerobic and anaerobic exercises in polluted air. Journal of Arak University of Medical Sciences. 2010;13(2):91-9. [Persian].

20. Spathopoulos D, Paraskakis E, Trypsianis G, Tsalkidis A, Arvanitidou V, Emporiadou M, et al. The effect of obesity on pulmonary lung function of school aged children in Greece. Pediatr Pulmonol. 2009;44(3):273-80. doi: 10.1002/ ppul.20995.

21. Shore SA, Johnston RA. Obesity and asthma. Pharmacol Ther. 2006;110(1):83-102. doi: 10.1016/j.pharmthera.2005.10.002.

22. de Oliveira PD, Wehrmeister FC, Horta BL, Pérez-Padilla R, de França GVA, Gigante DP, et al. Visceral and subcutaneous abdominal adiposity and pulmonary function in 30-year-old adults: a cross-sectional analysis nested in a birth cohort. BMC Pulm Med. 2017;17(1):157. doi: 10.1186/s12890-017-0510-7.

23. Tkacova R. Systemic inflammation in chronic obstructive pulmonary disease: may adipose tissue play a role? review of the literature and future perspectives. Mediators Inflamm. 2010;2010:585989. doi: 10.1155/2010/585989.

24. Sutherland TJ, Goulding A, Grant AM, Cowan JO, Williamson A, Williams SM, et al. The effect of adiposity measured by dualenergy X-ray absorptiometry on lung function. Eur Respir J. 2008;32(1):85-91. doi: 10.1183/09031936.00112407.

25. Procaccini C, Jirillo E, Matarese G. Leptin as an immunomodulator. Mol Aspects Med. 2012;33(1):35-45. doi: 10.1016/j.mam.2011.10.012.

26. Thorleifsson SJ, Margretardottir OB, Gudmundsson G, Olafsson I, Benediktsdottir B, Janson C, et al. Chronic airflow obstruction and markers of systemic inflammation: results from the BOLD study in Iceland. Respir Med. 2009;103(10):1548-53. doi: 10.1016/j.rmed.2009.04.005.

27. Parastesh. M, Heidarianpour a, Saremi A. The Effect of 12 weeks of aerobic training on lung function and serum leptin levels in obese men. Journal of Ilam University of Medical Sciences. 2014;22(1):139-46. [Persian].

28. Sierra-Johnson J, Romero-Corral A, Somers VK, Olson LJ, Johnson BD. Leptin, a novel predictor of lung function in heart failure. Chest. 2008;134(2):346-50. doi: 10.1378/ chest.07-2751.

29. Shore SA. Obesity and asthma: possible mechanisms. J Allergy Clin Immunol. 2008;121(5):1087-93. doi: 10.1016/j. jaci.2008.03.004.

30. Vogiatzis I. Strategies of muscle training in very severe COPD patients. Eur Respir J. 2011;38(4):971-5. doi: 10.1183/09031936.00075011. 